\title{
Crystal structure of the low-temperature modification of trirubidium heptaarsenide, $\mathbf{L T}-\mathbf{R b}_{3} \mathbf{A s}_{7}$
}

\author{
W. Hönle*.I J. Buresch ${ }^{\mathrm{I}}$, J. Wolf ${ }^{\mathrm{II}}$, K. Peters ${ }^{\mathrm{U}}$, J.-H. Chang ${ }^{\text {II }}$ and H. G. von Schnering ${ }^{\mathrm{II}}$ \\ 1 Max-Planck-Institut für Chemische Physik fester Stoffe. Nöthnitzerstr. 40. D-01187 Dresden. Germany \\ "Max-Planck-Institut für Festkörperforschung, Heisenbergstr. 1, D-70569 Stuttgart. Germany
}

Received September 17, 2002, accepted and available on-line October 17, 2002; CSD-No. 409652

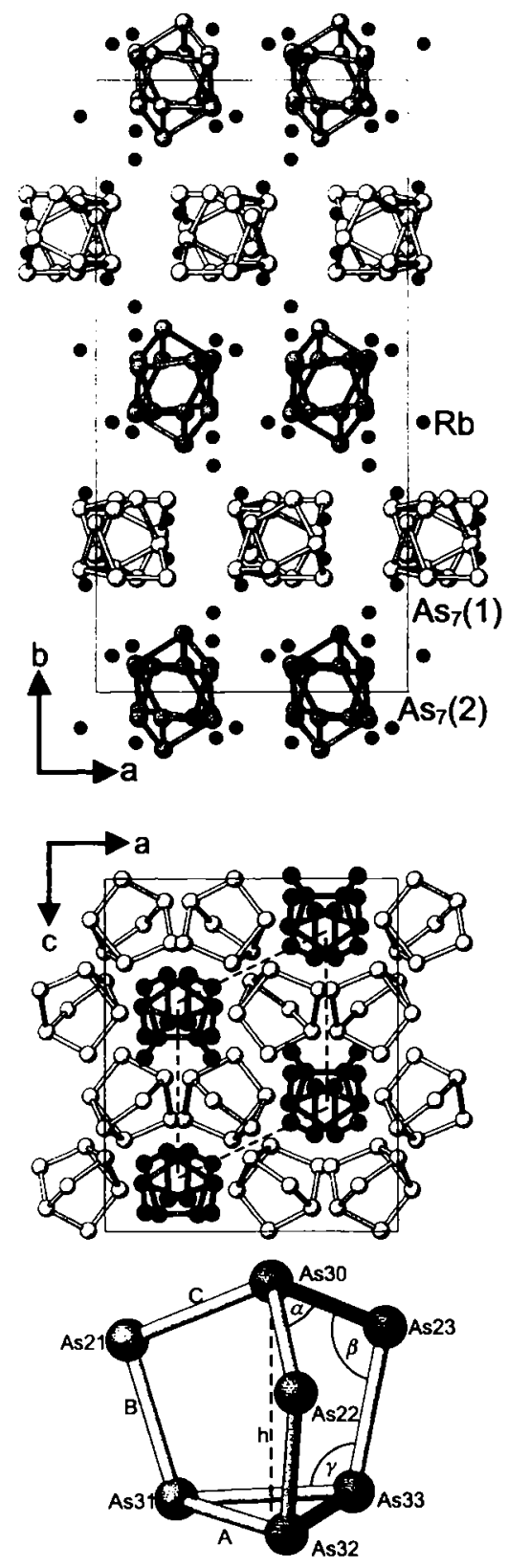

Abstract

As $7 \mathrm{Rb}_{3}$, orthorhombic, $P b c a$ (No. 61), $a=13.194(3) \AA$, $b=25.878(5) \AA, c=15.881(3) \AA, V=5422.3 \AA^{3}, Z=16$, $\left.R_{\mathrm{gt}}(F)\right)=0.082, w R_{\mathrm{obs}}\left(F^{2}\right)=0.140, w R_{\mathrm{all}}\left(F^{2}\right)=0.320, T=293 \mathrm{~K}$.

\section{Source of material}

Light crimson-red $\mathrm{Rb}_{3} \mathrm{As}_{7}$ (optical band gap $2.1 \mathrm{eV}$ ) is formed by reaction of stoichiometric mixtures of the elements in welded $\mathrm{Nb}$-tubes $(12 \mathrm{~h}$ up to $873 \mathrm{~K}, 36 \mathrm{~h}$ at $873 \mathrm{~K}$, down to $293 \mathrm{~K}$ within $36 \mathrm{~h})[1,2]$. The transformation to HT-Rb3 As7 takes place at $608 \mathrm{~K}$. The compound is very sensitive to hydrolysis and oxidation, and evaporates congruent dissociatively above $900 \mathrm{~K}$ in dynamic vacuum.

\section{Experimental details}

The relatively large residual factor reflects the poor crystal quality caused by the low transition temperature from HT to LT phase (at about $608 \mathrm{~K}$ ). Also, the absorption correction obviously does not account for the remaining differences in the $U_{\mathrm{ij}}$ values.

\section{Discussion}

LT-Rb 3 As7 forms a new $o P 160$ structure type. The arrangement of the two crystallographically independent Zintl anions (As7) ${ }^{3-}$ (upper and middle figures) represents a hierarchical cluster-replacement structure [3] of the hexagonal $\alpha$-La type. For a direct comparison, the $c a b$ setting must be chosen (in the $a b c$ setting: $a_{\text {orth }} \cong$ $c_{\text {orth }} \sqrt{3} / 2 \equiv a_{\text {hex }}, b_{\text {orth }} \equiv c_{\text {hex }}$ ). The quasi-hexagonal unit cell is emphasized by broken lines in the middle figure.

The mean As-As bond lengths and the bond angles $(\mathrm{A}=2.510 \AA$, $\mathrm{B}=2.343 \AA, \mathrm{C}=2.387 \AA, \alpha=101.4^{\circ}, \beta=99,6^{\circ}, \gamma=104,7^{\circ}, \mathrm{h}=$ $3.317 \AA$; lower figure) are characteristic for strongly ionic nortricyclenes with $\mathrm{A}>\mathrm{C}>\mathrm{B}, \alpha>\beta$ and $\mathrm{Q}=\mathrm{h} / \mathrm{A}=1.32 \AA[4,5]$. The $\mathrm{Rb}^{+}$cations connect $4-6(\mathrm{As} 7)^{3-}$ anions $(d(\mathrm{Rb}-\mathrm{As})=3.43 \AA-$ $4.20 \AA$ ) and show the preformation of $\mathrm{Rb}_{3} \mathrm{As}_{7}$ molecules $[5,6]$ and especially $\left(\mathbf{R b}_{2} \mathrm{As} 7-\mathbf{R b}-\mathbf{R} \mathbf{b}_{2} \mathrm{As}_{7}\right)^{-}$subunits. The first order phase transition to the plastically crystalline cubic HT-Rb 3 As $_{7}$ [6] (hierarchical cluster-replacement $\mathrm{Li}_{3} \mathrm{Bi}$ structure) requires considerable rearrangements.

Table 1. Data collection and handling.

\section{Crystal:}

Wavelength:

$\mu$ :

Diffractometer, scan mode: $2 \theta_{\max }:$

$N(h k l)_{\text {measured, }} N(h k l)_{\text {unique: }}$ Criterion for $I_{\text {obs, }} N(h k l)_{g 1:}$ $N$ (param)refined: Programs: light crimson-red polyhedron size $0.2 \times 0.3 \times 0.3 \mathrm{~mm}$ Mo $K_{a}$ radiation $(0.71073 \AA)$ $276.90 \mathrm{~cm}^{-1}$

Siemens P1, $\omega$ $50^{\circ}$ 4777,4777 $I_{\text {obs }}>2 \sigma\left(I_{\mathrm{obs}}\right), 1308$

182 SHELXS-97 [7], SHELXI-97 [8] ATOMS [9], DIFABS [10]

\footnotetext{
* (Correspondence author (e-mail: hoenle@cpfs.mpg.de)
} 
Table 2. Atomic coordinates and displacement parameters (in $\AA^{2}$ ).

\begin{tabular}{|c|c|c|c|c|c|c|c|c|c|c|}
\hline Atom & Site & $x$ & $y$ & $z$ & $U_{11}$ & $U_{22}$ & $U_{33}$ & $U_{12}$ & $U_{13}$ & $U_{23}$ \\
\hline $\operatorname{As}(301)^{a}$ & $8 c$ & $0.2056(5)$ & $0.2593(3)$ & $0.0655(7)$ & $0.040(4)$ & $0.042(4)$ & $0.045(6)$ & $0.006(3)$ & $-0.004(5)$ & $0.003(5)$ \\
\hline As(211) & $8 c$ & $0.2288(6)$ & $0.3139(3)$ & $0.1836(7)$ & $0.052(5)$ & $0.036(4)$ & $0.056(7)$ & $-0.012(3)$ & $0.001(5)$ & $0.019(5)$ \\
\hline As(221) & $8 c$ & $0.1308(6)$ & $0.1853(3)$ & $0.1293(7)$ & $0.060(5)$ & $0.025(4)$ & $0.050(7)$ & $0.003(3)$ & $-0.022(5)$ & $-0.015(4)$ \\
\hline $\operatorname{As}(231)$ & $8 c$ & $0.0575(6)$ & $0.2981(3)$ & $0.0023(6)$ & $0.046(4)$ & $0.041(4)$ & $0.030(5)$ & $0.005(3)$ & $0.000(5)$ & $0.007(4)$ \\
\hline $\operatorname{As}(311)$ & $8 c$ & $0.0651(6)$ & $0.3132(3)$ & $0.2405(6)$ & $0.063(5)$ & $0.041(4)$ & $0.040(6)$ & $0.014(4)$ & $-0.017(5)$ & $0.001(5)$ \\
\hline As (321) & $8 c$ & $-0.0009(6)$ & $0.2242(3)$ & $0.2072(6)$ & $0.046(4)$ & $0.041(4)$ & $0.052(7)$ & $-0.010(3)$ & $-0.020(5)$ & $0.014(5)$ \\
\hline $\operatorname{As}(302)$ & $8 c$ & $0.3163(5)$ & $0.5414(3)$ & $-0.0485(6)$ & $0.049(4)$ & $0.027(4)$ & $0.040(6)$ & $0.005(3)$ & $-0.010(5)$ & $-0.013(4)$ \\
\hline As $(212)$ & $8 c$ & $0.1373(5)$ & $0.5301(3)$ & $-0.0531(6)$ & $0.035(4)$ & $0.050(4)$ & $0.037(6)$ & $0.012(3)$ & $0.012(5)$ & $-0.003(5)$ \\
\hline $\operatorname{As}(222)$ & $8 c$ & $0.3661(5)$ & $0.5357(3)$ & $-0.1922(7)$ & $0.036(4)$ & $0.054(5)$ & $0.012(5)$ & $0.004(4)$ & $-0.004(5)$ & $0.012(5)$ \\
\hline $\operatorname{As}(232)$ & $8 c$ & $0.3644(5)$ & $0.4576(3)$ & $0.0083(6)$ & $0.046(4)$ & $0.040(4)$ & $0.036(6)$ & $0.000(3)$ & $-0.007(5)$ & $0.013(5)$ \\
\hline $\operatorname{As}(312)$ & $8 c$ & $0.1272(6)$ & $0.4550(3)$ & $-0.1360(6)$ & $0.047(5)$ & $0.051(5)$ & $0.034(6)$ & $-0.011(4)$ & $0.010(5)$ & $0.001(5)$ \\
\hline As (322) & $8 c$ & $0.2858(6)$ & $0.4572(3)$ & $-0.2253(6)$ & $0.049(4)$ & $0.044(4)$ & $0.039(6)$ & $0.005(3)$ & $-0.002(5)$ & $-0.011(5)$ \\
\hline As(332) & $8 c$ & $0.2825(6)$ & $0.4057(3)$ & $-0.0926(6)$ & $0.060(5)$ & $0.025(4)$ & $0.042(6)$ & $0.002(3)$ & $-0.002(5)$ & $-0.004(4)$ \\
\hline$R b(1)$ & $8 c$ & $0.1260(5)$ & $0.5832(2)$ & $-0.2545(7)$ & $0.052(4)$ & $0.032(3)$ & $0.052(6)$ & $-0.005(3)$ & $0.002(5)$ & $0.002(5)$ \\
\hline $\mathbf{R b}(3)$ & $8 c$ & $0.1153(5)$ & $0.4249(3)$ & $0.0885(6)$ & $0.051(5)$ & $0.048(4)$ & $0.035(5)$ & $0.010(3)$ & $-0.020(5)$ & $0.007(4)$ \\
\hline $\mathbf{R b}(4)$ & $8 c$ & $0.2303(5)$ & $0.2823(3)$ & $-0.1627(6)$ & $0.053(5)$ & $0.039(4)$ & $0.051(6)$ & $-0.005(3)$ & $0.003(5)$ & $-0.012(4)$ \\
\hline Rb(5) & $8 c$ & $0.3764(6)$ & $0.1297(3)$ & $0.1065(8)$ & $0.070(6)$ & $0.061(5)$ & $0.085(9)$ & $-0.008(4)$ & $0.041(7)$ & $0.005(6)$ \\
\hline $\mathrm{Rb}(6)$ & $8 c$ & $-0.0351(6)$ & $0.1750(3)$ & $-0.0445(7)$ & $0.082(6)$ & $0.049(4)$ & $0.058(7)$ & $-0.027(4)$ & $-0.004(6)$ & $0.003(5)$ \\
\hline
\end{tabular}

a: labelling of the As atoms As(ijk) with $i=$ homoatomic connectivity, $j=$ position in cluster (lower figure), $k=$ number of As7 cluster (upper figure).

\section{References}

1. Wolf, J.: Germyl- und Stannylsubstituierte Heptaarsane(3) und Undecaarsane(3) als Reaktionsprodukte binärer Metallarsenide sowie über die neuartigen Komplexe, $\eta^{8}$-Cyclooctaarsenidoniobat(V)(3-) $\left[\mathrm{NbAs}_{8}\right]^{3-}$ und catena-Diarsasilikat ${ }_{\infty}^{1}\left[\mathrm{SiAs}_{2}\right]^{2-}$. Dissertation, Universität Stuttgart, 1986.

2. Buresch, J.: Binäre und ternäre Arsenide, Antimonide und ArsenidAntimonide der Alkalimetalle mit den Anionen $\mathrm{X}^{3-}, \mathrm{X}_{3}^{5-}, \mathrm{X}_{4}^{-5-},{ }_{\infty}^{1}[\mathrm{X}]$, $\mathrm{X}_{7}{ }^{3-}$ und $\mathrm{X}_{7}^{5-}$. Dissertation, Universität Stuttgart, 1996.

3. Carrillo-Cabrera, W.: Caroca-Canales, N.; von Schnering, H. G.:

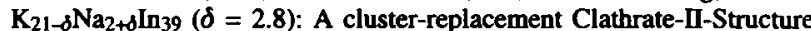
with an Alkali Metal M136-Network. Z. Anorg. Allg. Chem. 620 (1994) 247-257.

4. Hönle, W.; von Schnering, H. G.: Die Strukturen der Hepta-HeteroNortricyclene $\mathrm{P}_{7}\left(\mathrm{Sime}_{3}\right)_{3}$ und $\mathrm{P}_{4}\left(\mathrm{Sime}_{2}\right)_{4}$. Z. Anorg. Allg. Chem. 440 (1978) 171-182.
5. von Schnering, H. G.; Hönle, W.: Bridging Chasms with Polyphosphides, Chem. Rev. 88 (1988) 243-273.

6. Hönle, W.; von Schnering, H. G., Somer, M.: Strukturen von Heptaarseniden der Alkalimetalle und deren Solvaten. Z. Kristallogr. 174 (1986) 82-83.

7. Sheldrick, G. M.: Phase Annealing in SHELX-90: Direct Methods for Larger Structures. Acta Crystallogr. A46 (1990) 467-473.

8. Sheldrick, G. M.: SHELXL-97. Program for the Refinement of Crystal Structures. University of Göttingen, Germany 1997.

9. Dowty, E.: ATOMS 5.1. A Complete Program for Displaying Atomic Structures. By Shape software, 521 Hidden Valley Road, Kingsport, TN 37663, USA 2000.

10. Walker, N.; Stuart, D.: An Empirical Method for Correcting Diffractometer Data for Absorption Effects. Acta Crystallogr. A39 (1993) 159-166. 